International Mathematical Forum, 2, 2007, no. 16, 781 - 802

\title{
Regular Polygonal Numbers and Generalized Pell Equations
}

\author{
CHU Wenchang \\ Dipartimento di Matematica \\ Università degli Studi di Lecce \\ Lecce-Arnesano, P.O.Box 193, 73100 Lecce, Italy \\ tel $39+0832+297409, \quad$ fax $39+0832+297594$ \\ chu.wenchang@unile.it
}

\begin{abstract}
In the eighteenth century, both square numbers and triangular numbers were investigated by Euler and Goldbach (1742), who determined the recurrence relations satisfied by the sequence and established the general formulae explicitly. It seems to the author that the topics around this subject have not been touched in mathematical literature. As the first attempt to explore it, this work will present a systematic procedure to deal with the problem. For the regular $(\lambda, \mu)$-polygonal numbers, the corresponding Diophantine equations will be reduced to the generalized Pell equations. Then solutions of the associated Pell equations will essentially enable us to resolve the problem. By means of Computer Algebra, the recurrence relations and generating functions satisfied by $(\lambda, \mu)$-polygonal numbers can be recovered systematically. As exemplification, the results on the first twenty regular $(\lambda, \mu)$-polygonal sequences will be presented in details.
\end{abstract}

Mathematics Subject Classification: Primary 11Y50, Secondary 11Y55

Keywords: Polygonal number, Diophantine equation, Generalized Pell equation, Recurrence relation, Generating function

\section{InTRODUCTION AND NotATION}

In the treatise Polygonal Numbers, Diophantus quoted the definition of polygonal numbers (cf. [1, pp. 1-3] for reference) due to Hypsicles (around 175 B.C.): "If there are as many numbers as we please beginning with one and increasing by the same common difference, then when the common difference is one, the sum of all the terms is a triangular number; when 2, a square; when 3 , a pentagonal number. And the number of the angles is called after the number exceeding the common difference by 2 , and the side after the number of terms including 1". Given therefore an arithemetical progression with the first term 1 and the common difference $\lambda-2$, the sum of $n$ terms is the regular $n$-th $\lambda$-gonal number $p_{\lambda}(n)$. 
From this definition, we can compute the regular $n$-th $\lambda$-gonal number $p_{\lambda}(n)$ explicitly as follows:

$$
p_{\lambda}(n)=\sum_{k=0}^{n-1}\{1+(\lambda-2) k\}=n+(\lambda-2)\left(\begin{array}{c}
n \\
2
\end{array}\right)
$$

where $n=1,2, \cdots$. Hence the set of all the regular $\lambda$-polygonal numbers is determined by

$$
\mathcal{P}_{\lambda}=\left\{n+(\lambda-2)\left(\begin{array}{c}
n \\
2
\end{array}\right) \mid n=1,2, \cdots\right\}
$$

Triangular numbers and square numbers are respectively given by

$$
\mathcal{P}_{3}=\left\{\left(\begin{array}{c}
n+1 \\
2
\end{array}\right) \mid n=1,2, \cdots\right\} \quad \text { and } \quad \mathcal{P}_{4}=\left\{n^{2} \mid n=1,2, \cdots\right\}
$$

They can be geometrically represented by the following figures:
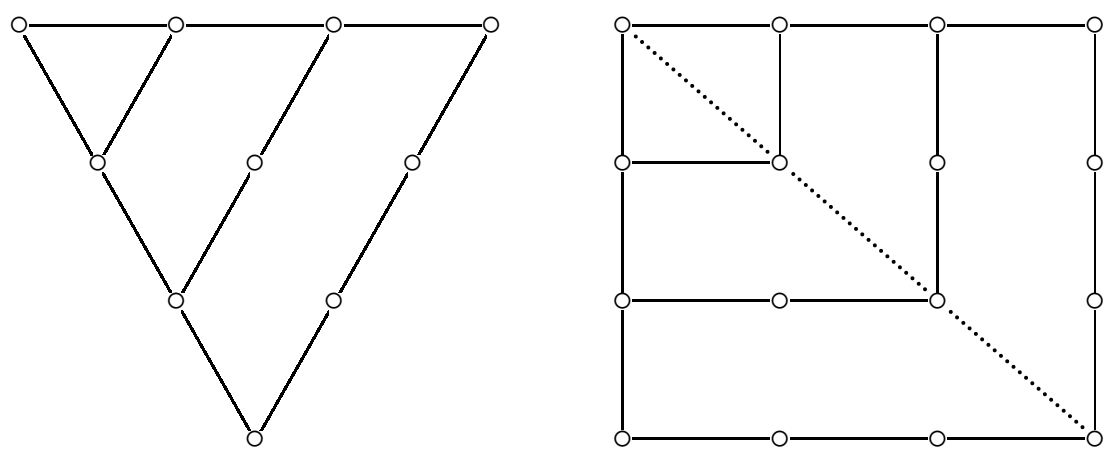

In general, the regular polygonal numbers can be generated recursively as follows. For $p_{\lambda}(n)$, fix with $\lambda$ the number of sides of the polygons, called $\lambda$-polygons, and with $n$ the side-length minus one. Conventionally one fixes $p_{\lambda}(1)=1$ because the regular $\lambda$-polygon with side length equal to zero reduces to one point. Based on a $\lambda$-polygon with side-length equal to $n-1$, we can construct the next polygon with the side-length equal to $n$, extending by unit the two base sides which cross at the starting point and then adding $\lambda-2$ new sides parallel with the remaining sides. During this construction, we have added $1+n(\lambda-2)$ new points to the polygon on the new sides. This procedure can be illustrated by the following figure of hexagons: 


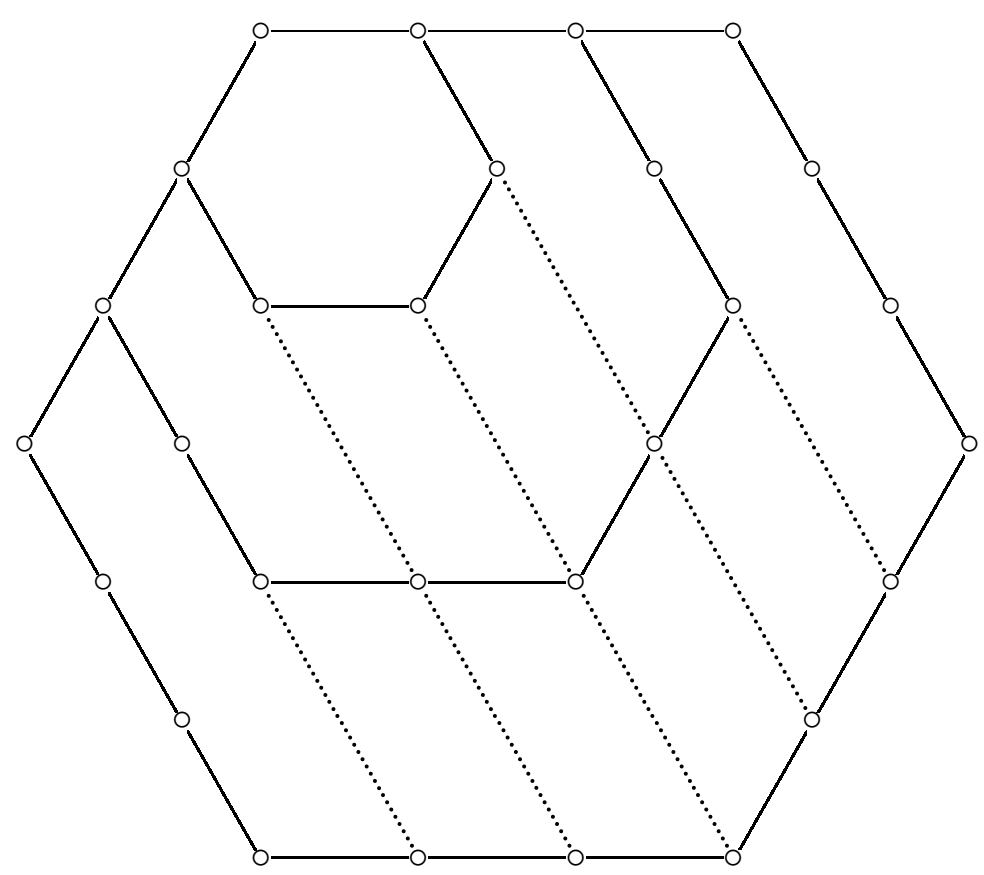

Therefore we have the following recurrence relation

$$
\begin{aligned}
& p_{\lambda}(1+n)=1+n(\lambda-2)+p_{\lambda}(n) \\
& \text { with } \quad p_{\lambda}(1)=1 \quad \text { and } \quad n=1,2, \cdots
\end{aligned}
$$

which is consistent with (1.1), derived from the arithemetical setting.

For $\lambda, \mu \in \mathbb{N}$ with $\lambda \neq \mu$ and $\lambda, \mu \geq 3$, we define the regular $(\lambda, \mu)$-polygonal numbers to be the natural numbers in the intersection set $\mathcal{P}_{\lambda} \cap \mathcal{P}_{\mu}$, which are both regular $\lambda$-polygonal and $\mu$-polygonal. They are characterized by natural number solutions $(x, y)$ of the Diophantine equation $p_{\lambda}(x)=p_{\mu}(y)$, written explicitly as

$$
x+(\lambda-2)\left(\begin{array}{l}
x \\
2
\end{array}\right)=y+(\mu-2)\left(\begin{array}{l}
y \\
2
\end{array}\right) \text {. }
$$

It is not hard to check that there is bijective correspondence between $\mathcal{P}_{\lambda} \cap \mathcal{P}_{\mu}$ and the solutions of $p_{\lambda}(x)=p_{\mu}(y)$ in natural numbers. In fact, for any $z \in$ $\mathcal{P}_{\lambda} \cap \mathcal{P}_{\mu}$, there exist exactly two natural numbers $x$ and $y$ such that $z=$ $p_{\lambda}(x)=p_{\mu}(y)$. Throughout the paper, the regular $(\lambda, \mu)$-polygonal numbers will be represented by the triples $(x, y ; z)$, where $z$ is both regular $\lambda$-gonal and $\mu$-gonal number with side-lengths equal to $x$ and $y$ respectively. 
For $\lambda=3$ and $\mu=4$, the first triangular-square numbers can be displayed in the following table:

\begin{tabular}{||c|c|c|c||}
\hline \hline$n$ & $x_{n}$ & $y_{n}$ & $z_{n}$ \\
\hline 0 & 1 & 1 & 1 \\
\hline 1 & 8 & 6 & 36 \\
\hline 2 & 49 & 35 & 1225 \\
\hline 3 & 288 & 204 & 41616 \\
\hline 4 & 1681 & 1189 & 1413721 \\
\hline 5 & 9800 & 6930 & 48024900 \\
\hline \hline
\end{tabular}

Euler observed in 1730 that the triangle-square numbers have the following form (cf. Dickson [1, p. 10]):

$$
\begin{array}{ll}
x_{n}=\frac{(3+2 \sqrt{2})^{n}+(3-2 \sqrt{2})^{n}}{4}-\frac{1}{2} \quad(n \in \mathbb{N}) \\
y_{n}=\frac{(3+2 \sqrt{2})^{n}-(3-2 \sqrt{2})^{n}}{4 \sqrt{2}} \quad(n \in \mathbb{N}) .
\end{array}
$$

Almost half century later, Euler (1778) proved that these are all the regular $(3,4)$-polygonal numbers (cf. Dickson [1, p. 16]) which also satisfy the following crossing recurrence relations of the first degree:

$$
\left.\begin{array}{l}
x_{1+m}=3 x_{m}+4 y_{m}+1 \\
y_{1+m}=3 y_{m}+2 x_{m}+1
\end{array}\right\} \quad x_{0}=y_{0}=1
$$

and the independent recurrence relations of the second degree

$$
\begin{aligned}
& x_{1+n}=6 x_{n}-x_{n-1}+2: \quad x_{0}=1, \quad x_{1}=8 \\
& y_{1+n}=6 y_{n}-y_{n-1}: \quad y_{0}=1, \quad y_{1}=6
\end{aligned}
$$

as well as the respective generating functions:

$$
\begin{aligned}
f(x) & :=\sum_{n=0}^{\infty} x^{n} x_{n}=\frac{1+x}{(1-x)\left(1-6 x+x^{2}\right)} \\
g(y) & :=\sum_{n=0}^{\infty} y^{n} y_{n}=\frac{1}{1-6 y+y^{2}} .
\end{aligned}
$$

Moreover, in 1742, there was a communication (see Dickson [1, pp. 10-11]) between Euler and Goldbach on the pentagon numbers

$$
\mathcal{P}_{5}=\left\{\frac{3 n^{2}-n}{2} \mid n=1,2, \cdots\right\}
$$

for which there holds the following celebrated pentagon number theorem due to Euler (cf. [2, §19.9]):

$$
\prod_{m=1}^{\infty}\left(1-q^{m}\right)=\sum_{n=-\infty}^{+\infty}(-1)^{n} q^{\frac{3 n^{2}-n}{2}} \quad \text { where }|q|<1 .
$$


The object of the paper is to determine all the regular $(\lambda, \mu)$-polygonal numbers. As preliminaries, we shall review basic facts about the Pell equations and generalized Pell equations in the next section. Then the Diophantine equation (1.4) will be reduced to generalized Pell equation in the third section. The classification, recurrence relations and generating functions of the regular $(\lambda, \mu)$-polygonal numbers will be investigated in the fourth section. By means of computer algebra, the fifth and the last section collects twenty examples of the regular $(\lambda, \mu)$-polygonal numbers, which presents a full coverage for the cases $3 \leq \lambda \neq \mu \leq 9$.

\section{Generalized Pell Equations}

In order to investigate the Diophantine equations on the regular $(\lambda, \mu)$ polygonal numbers, we review some basic results about Pell equations and generalized Pell equations. For details, the reader can refer to the books $[3$, $\S 10.9$ and $\S 11.5],[6, \S 6.2]$ and $[8, \S 7.8]$.

2.1. Pell equation. With $D$ being a non-perfect-square natural number, Pell equation

$$
u^{2}-D v^{2}=1
$$

admits always infinite solutions. They can be determined through continued fraction expansion of $\sqrt{D}$. If $(u, v)$ is the minimal positive solution, then all the non-negative solutions are given by

$$
u_{n} \pm v_{n} \sqrt{D}=\{u \pm v \sqrt{D}\}^{n}, \quad\left(n \in \mathbb{N}_{0}\right)
$$

which leads us to the following explicit formulas:

$$
\begin{aligned}
& u_{n}=\frac{1}{2}\left\{(u+v \sqrt{D})^{n}+(u-v \sqrt{D})^{n}\right\} \\
& v_{n}=\frac{1}{2 \sqrt{D}}\left\{(u+v \sqrt{D})^{n}-(u-v \sqrt{D})^{n}\right\} .
\end{aligned}
$$

In addition, the solutions $\left\{u_{n}, v_{n}\right\}$ satisfy the recurrence relations

$$
\left.\begin{array}{l}
u_{1+n}=2 u u_{n}-u_{n-1} \\
v_{1+n}=2 u v_{n}-v_{n-1}
\end{array}\right\} \quad \text { and } \quad\left\{\begin{array}{l}
u_{1+n}=u u_{n}+v v_{n} D \\
v_{1+n}=u v_{n}+v u_{n} .
\end{array}\right.
$$

2.2. Generalized Pell equation. For two integers $D$ and $N$ with $D$ being positive and non-perfect-square, the generalized Pell equation and the associated Pell equation are displayed as

$$
U^{2}-D V^{2}=N \quad \text { and } \quad u^{2}-D v^{2}=1
$$

If $(U, V)$ and $(u, v)$ are solutions respectively corresponding to the generalized Pell equation and the associated Pell equation, then

$$
(u U+v V D, u V+v U)
$$


derived from the product (cf. $[7, \S 58]$ )

$$
(u+v \sqrt{D}) \times(U+V \sqrt{D})=(u U+v V D)+(u V+v U) \sqrt{D}
$$

forms a new solution of the generalized Pell equation thanks to the relation

$$
(u U+v V D)^{2}-D(u V+v U)^{2}=\left(u^{2}-D v^{2}\right) \times\left(U^{2}-D V^{2}\right) .
$$

Two solutions $(U, V)$ and $\left(U^{\prime}, V^{\prime}\right)$ of the generalized Pell equation are said to be equivalent if there exists one solution $(u, v)$ of the associated Pell equation such that

$$
\left(U^{\prime}, V^{\prime}\right)=(u U+v V D, u V+v U) .
$$

Therefore all the solutions of the generalized Pell equation can be divided into equivalent classes, among which each class of solutions consist of doublesequences subject to the crossing recurrence relation of the first degree:

$$
\begin{aligned}
& U_{1+n}=u U_{n}+v V_{n} D \\
& V_{1+n}=u V_{n}+v U_{n}
\end{aligned}
$$

where $(u, v)$ is a solution of the associated Pell equation.

Now replacing $n$ by $n-1$, we can restate the crossing recurrences $(2.4 \mathrm{a}-2.4 \mathrm{~b})$ as

$$
\begin{aligned}
U_{n} & =u U_{n-1}+v V_{n-1} D \\
V_{n} & =u V_{n-1}+v U_{n-1} .
\end{aligned}
$$

Eliminating $V_{n-1}$ and $U_{n-1}$ from these two equations and noting that $u^{2}-$ $D v^{2}=1$, we obtain the following expressions

$$
\begin{aligned}
D v V_{n} & =u U_{n}-U_{n-1} \\
v U_{n} & =u V_{n}-V_{n-1} .
\end{aligned}
$$

Substituting them into $(2.4 \mathrm{a})$ and $(2.4 \mathrm{~b})$, we get the independent recurrence relations of the second degree

$$
\begin{aligned}
& U_{1+n}=2 u U_{n}-U_{n-1} \\
& V_{1+n}=2 u V_{n}-V_{n-1}
\end{aligned}
$$

which are satisfied by the equivalent class of solutions of the generalized Pell equation corresponding to the crossing recursions (2.4a-2.4b).

\section{Diophantine Equation $p_{\lambda}(x)=p_{\mu}(y)$}

For the Diophantine Equation $p_{\lambda}(x)=p_{\mu}(y)$, given explicitly by (1.4), this section will show how to reduce it canonically to the generalized Pell equation. 
Firstly, define two integer parameters:

$$
\begin{aligned}
& d(\lambda)=\operatorname{gcd}(\lambda-4,2 \lambda-4)=\left\{\begin{array}{lll}
1, & \lambda \equiv 1 & (\bmod 2) \\
2, & \lambda \equiv 2 & (\bmod 4) \\
4, & \lambda \equiv 0 & (\bmod 4)
\end{array}\right. \\
& c(\lambda, \mu)=\operatorname{gcd}\left\{d^{2}(\lambda)(\mu-2), d^{2}(\mu)(\lambda-2)\right\} .
\end{aligned}
$$

They allow us to rewrite the regular $\lambda$-polygonal numbers as

$$
\begin{aligned}
8 p_{\lambda}(x) & =4(\lambda-2)\left\{x-\frac{\lambda-4}{2 \lambda-4}\right\}^{2}-\frac{(\lambda-4)^{2}}{\lambda-2} \\
& =\frac{1}{\lambda-2}\{(2 \lambda-4) x-(\lambda-4)\}^{2}-\frac{(\lambda-4)^{2}}{\lambda-2} \\
& =\frac{d^{2}(\lambda)}{\lambda-2}\left\{\frac{2 \lambda-4}{d(\lambda)} x-\frac{\lambda-4}{d(\lambda)}\right\}^{2}-\frac{(\lambda-4)^{2}}{\lambda-2}
\end{aligned}
$$

and the Diophantine equation $p_{\lambda}(x)=p_{\mu}(y)$ as

$$
\begin{aligned}
\frac{(\lambda-\mu)(\lambda \mu-2 \lambda-2 \mu)}{c(\lambda, \mu)} & =\frac{(\mu-2) d^{2}(\lambda)}{c(\lambda, \mu)}\left\{\frac{2 \lambda-4}{d(\lambda)} x-\frac{\lambda-4}{d(\lambda)}\right\}^{2} \\
& -\frac{(\lambda-2) d^{2}(\mu)}{c(\lambda, \mu)}\left\{\frac{2 \mu-4}{d(\mu)} y-\frac{\mu-4}{d(\mu)}\right\}^{2} .
\end{aligned}
$$

Secondly, with $\mathbf{B}$ and $\mathbf{M}$-coefficients being defined respectively by

$$
\begin{aligned}
B(\lambda, \mu) & =\frac{(\mu-2) d^{2}(\lambda)}{c(\lambda, \mu)} \\
M(\lambda, \mu) & =\frac{(\lambda-\mu)(\lambda \mu-2 \lambda-2 \mu)}{c(\lambda, \mu)}
\end{aligned}
$$

we have evidently $\operatorname{gcd}\{B(\lambda, \mu), B(\mu, \lambda)\}=1$ in view of (3.1b). Under the linear transformation

$$
\mathcal{S}:\left\{\begin{array}{l}
X=\frac{2 \lambda-4}{d(\lambda)} x-\frac{\lambda-4}{d(\lambda)} \\
Y=\frac{2 \mu-4}{d(\mu)} y-\frac{\mu-4}{d(\mu)}
\end{array}\right.
$$

the Diophantine equation (1.4) is equivalent to the following

$$
B(\lambda, \mu) X^{2}-B(\mu, \lambda) Y^{2}=M(\lambda, \mu) .
$$

Observe that $B(\lambda, \mu)$ is an integer on account (3.1a) and (3.1b). For the same reason, we can check that $M(\lambda, \mu)$ is an integer either, just specifying (3.2a-3.2b) by $x=y=1$, which is the "universal solution" of (1.4).

Lastly, applying the square-free factorization

$$
B(\lambda, \mu)=p(\lambda, \mu) q^{2}(\lambda, \mu)
$$


and introducing $\mathbf{D}$ and $\mathbf{N}$-coefficients:

$$
\begin{aligned}
& D(\lambda, \mu)=p(\lambda, \mu) p(\mu, \lambda) \\
& N(\lambda, \mu)=p(\lambda, \mu) M(\lambda, \mu)
\end{aligned}
$$

we can restate (3.5) as the generalized Pell equation:

$$
U^{2}-D(\lambda, \mu) V^{2}=N(\lambda, \mu)
$$

where we have further introduced the linear transformation

$$
\mathcal{T}:\left\{\begin{array}{l}
U=p(\lambda, \mu) q(\lambda, \mu) X \\
V=q(\mu, \lambda) Y .
\end{array}\right.
$$

Summing up, the Diophantine equation (1.4) on regular $(\lambda, \mu)$-polygonal numbers has been reduced to the generalized Pell equation (3.8) under the composite transformation

$$
\Omega[\lambda, \mu]=\mathcal{T} \circ \mathcal{S}:(x, y) \longrightarrow\{U(x), V(y)\}
$$

explicitly given by:

$$
\Omega[\lambda, \mu]:\left\{\begin{array}{l}
U(x)=\left\{\frac{2 \lambda-4}{d(\lambda)} x-\frac{\lambda-4}{d(\lambda)}\right\} p(\lambda, \mu) q(\lambda, \mu) \\
V(y)=\left\{\frac{2 \mu-4}{d(\mu)} y-\frac{\mu-4}{d(\mu)}\right\} q(\mu, \lambda)
\end{array}\right.
$$

whose inverse transformation reads as

$$
\Omega^{\prime}[\lambda, \mu]:\left\{\begin{array}{l}
x=\frac{d(\lambda) U+(\lambda-4) p(\lambda, \mu) q(\lambda, \mu)}{(2 \lambda-4) p(\lambda, \mu) q(\lambda, \mu)} \\
y=\frac{d(\mu) V+(\mu-4) q(\mu, \lambda)}{(2 \mu-4) q(\mu, \lambda)} .
\end{array}\right.
$$

The antisymmetry $M(\lambda, \mu)=-M(\mu, \lambda)$ allows us to choose always the nonnegative parameter $N(\mu, \lambda)=p(\mu, \lambda) M(\mu, \lambda)$ by interchanging the positions of $\lambda$ and $\mu$.

Remark: Recalling that $\left(x_{0}, y_{0}\right)=(0,0)$ and $\left(x_{1}, y_{1}\right)=(1,1)$ are always solutions of (1.4), we affirm that $\{U(0), V(0)\}$ and $\{U(1), V(1)\}$ deduced from the composite transformation $\Omega[\lambda, \mu]$ are always solutions of the generalized Pell equation (3.8).

\section{Regular $(\lambda, \mu)$-Polygonal Numbers}

When the $D$-coefficient defined by (3.7a) is equal to one, we say that the corresponding Diophantine equation (1.4) is reducible. In that case, for $(\lambda, \mu) \neq$ $(3,6)$ or $(6,3)$, equation (1.4) would have finitely many solutions (see $\S 4.1$ and $\S 4.2$ ). Otherwise, the Diophantine equation (1.4) corresponding to $D \neq 1$ is said to be irreducible and the generalized Pell equation (3.8) has infinitely many solutions (see $\S 4.3$ below). Therefore, the original Diophantine equation (1.4) on regular $(\lambda, \mu)$-polygonal numbers has infinitely many solutions 
too. These solutions can be divided into equivalent classes and the solutions in each class can be determined by recurrence relations and accordingly generating functions.

4.1. Triangular and hexagonal numbers. Consider the extreme case of (3.8) when $N(\lambda, \mu)=0$, or $M(\lambda, \mu)=0$ equivalently, iff

$$
\lambda \mu-2 \lambda-2 \mu=0
$$

which may be reformulated as

$$
(\lambda-2)(\mu-2)=4 .
$$

Under the restrictions $\lambda \neq \mu \geq 3$, there is a unique solution $(\lambda, \mu)=(3,6)$. In this case, we have $D(3,6)=1$ and the equations (3.8) and (3.10) reduce to

$$
U^{2}-V^{2}=0: \quad \Omega[3,6]\left\{\begin{array}{l}
U=2 x+1 \\
V=4 y-1
\end{array}\right.
$$

which can be written explicitly as

$$
(U+V) \times(U-V)=0 \rightleftharpoons(x+2 y) \times(1+x-2 y)=0 .
$$

On account of solutions in natural numbers, we can write explicitly the solutions as:

$$
\left.\begin{array}{l}
x_{n}=2 n-1 \\
y_{n}=n
\end{array}\right\} \quad(n \in \mathbb{N})
$$

\begin{tabular}{||c|c|c|c||}
\hline \hline$n$ & $x_{n}$ & $y_{n}$ & $z_{n}$ \\
\hline 1 & 1 & 1 & 1 \\
\hline 2 & 3 & 2 & 6 \\
\hline 3 & 5 & 3 & 15 \\
\hline 4 & 7 & 4 & 28 \\
\hline 5 & 9 & 5 & 45 \\
\hline \hline
\end{tabular}

This means that all the hexagon numbers are also triangle numbers. This is the only reducible case where the equation (1.4) admits infinitely many solutions.

4.2. Reducible cases: Finite solutions. When $D(\lambda, \mu)=p(\lambda, \mu) p(\mu, \lambda)$ is a perfect square number, then $D(\lambda, \mu)=1$ thanks to the square free factorization. In this case, equation (3.8) reduces to

$$
(U+V) \times(U-V)=N(\lambda, \mu) .
$$

This equation can be resolved by factorizing $N(\lambda, \mu)$ into two integers and therefore has only finitely many solutions for $N(\lambda, \mu) \neq 0$, which is equivalent to $(\lambda, \mu) \neq(3,6)$ and $(6,3)$.

In view of $(3.3 \mathrm{a}),(3.6)$ and $(3.7 \mathrm{a})$, we have

$$
D(\lambda, \mu)=\frac{B(\lambda, \mu) B(\mu, \lambda)}{q^{2}(\lambda, \mu) q^{2}(\mu, \lambda)}=\frac{(\lambda-2)(\mu-2) d^{2}(\lambda) d^{2}(\mu)}{c^{2}(\lambda, \mu) q^{2}(\lambda, \mu) q^{2}(\mu, \lambda)} .
$$

Therefore $(\lambda-2)(\mu-2)$ must be a perfect square number. In this case, the Diophantine equation (1.4) has only finitely many solutions with $(\lambda, \mu) \neq(3,6)$ and $(6,3)$. 
Here we present a couple of examples to show the reducible cases.

When $(\lambda, \mu)=(11,6)$, both $\lambda-2=9$ and $\mu-2=4$ are perfect square numbers. The Diophantine equation (1.4) reads as

$$
x(9 x-7)=2 y(2 y-1)
$$

and the reduced Pell equation (3.8) becomes

$$
u^{2}-v^{2}=40: \quad \Omega[11,6]\left\{\begin{array}{l}
u=18 x-7 \\
v=12 y-3
\end{array}\right.
$$

which has only the solutions $( \pm 7, \pm 3)$ and $( \pm 11, \pm 9)$. Among these solutions, only $(11,9)$ gives natural numbers $(x, y)=(1,1)$ under the corresponding inverse transformation $\Omega^{\prime}[11,6]$ defined by (3.11). Therefore we have the only trivial regular $(11,6)$-polygonal number $(1,1)$.

For $(\lambda, \mu)=(29,5)$, neither $\lambda-2=27$ nor $\mu-2=3$ is perfect square number. But their product 81 is a perfect square number. The corresponding Diophantine equation (1.4) becomes

$$
x(27 x-25)=y(3 y-1)
$$

and the reduced Pell equation (3.8) reads as

$$
u^{2}-v^{2}=616: \quad \Omega[29,5]\left\{\begin{array}{l}
u=54 x-25 \\
v=18 y-3 .
\end{array}\right.
$$

It has only the solutions $( \pm 25, \pm 3),( \pm 29, \pm 15),( \pm 79, \pm 75)$ and $( \pm 155, \pm 153)$. Among these solutions, only $(29,15)$ gives natural numbers $(x, y)=(1,1)$ under the corresponding inverse transformation $\Omega^{\prime}[29,5]$ defined by (3.11). Therefore we have again in this case the only trivial regular $(29,5)$-polygonal number $(1,1)$.

4.3. Irreducible cases: Infinite solutions. When $(\lambda-2)(\mu-2)$ is not a perfect square number, the generalized Pell equation (3.8) is not reducible. Recalling that (3.8) has two universal solutions $\{U(0), V(0)\}$ and $\{U(1), V(1)\}$, we deduce that there exists at least one equivalent class of solutions for (3.8). Hence there are infinitely many solutions for the irreducible generalized Pell equation (3.8). These solutions can be classified, according to any fixed solution $(u, v)$ of the associated Pell equation, into equivalent classes, each of which satisfies recurrences:

$$
\left.\begin{array}{l}
U_{1+n}=u U_{n}+v V_{n} D \\
V_{1+n}=u V_{n}+v U_{n}
\end{array}\right\} \quad \text { and } \quad\left\{\begin{array}{l}
U_{1+n}=2 u U_{n}-U_{n-1} \\
V_{1+n}=2 u V_{n}-V_{n-1}
\end{array}\right.
$$

In what follows, we will determine the recurrence relations satisfied by the solutions of the Diophantine equation (1.4) on the regular $(\lambda, \mu)$-polygonal numbers and the corresponding generating functions. 
4.4. Recurrence relations. All the solutions of the Diophantine equation (1.4) turn, under $\Omega$-transform, into solutions of the generalized Pell equation

$$
U^{2}-D(\lambda, \mu) V^{2}=N(\lambda, \mu)
$$

Instead, each equivalent class of solutions of the generalized Pell equation obey crossing recursions (2.4a-2.4b), which are converted, under the inverse transform $\Omega^{\prime}$, into rational solutions of the original Diophantine equation (1.4). It is not difficult to check that the latter obey again crossing recursions, which can be figured out, by substituting the transformation (3.10) into the crossing recurrence relations (2.4a-2.4b), as follows:

$$
\begin{aligned}
& x_{1+n}=u x_{n}+v H(\lambda, \mu) y_{n}+E(\lambda, \mu \mid u, v) \\
& y_{1+n}=u y_{n}+v H(\mu, \lambda) x_{n}+E(\mu, \lambda \mid u, v)
\end{aligned}
$$

where

$$
\begin{aligned}
& H(\lambda, \mu)=\sqrt{\frac{\mu-2}{\lambda-2} D(\lambda, \mu)}=\frac{d(\mu) p(\lambda, \mu) q(\lambda, \mu)}{d(\lambda) q(\mu, \lambda)} \\
& E(\lambda, \mu \mid u, v)=\frac{4-\lambda}{4-2 \lambda}(1-u)-H(\lambda, \mu) \frac{4-\mu}{4-2 \mu} v
\end{aligned}
$$

In order to determine the crossing recurrence relations satisfied by the integer solutions of the Diophantine equation (1.4) on the regular $(\lambda, \mu)$-polygonal numbers, it is enough to find out the minimum $(u, v)$ among the solutions of the associated Pell-equation (2.1) such that (4.2a-4.2b) have integer coefficients.

Since $(1,1)$ is always a solution of the Diophantine equation $(1.4)$, we assert that any class of equivalent solutions must have the initial values $\left(x_{0}, y_{0}\right)$ with $x_{0}$ being from 1 to the sum of coefficients of (4.2a). Therefore the number of equivalent classes of the solutions corresponding to the solution $(u, v)$ of the associated Pell equation is less than $u+v H(\lambda, \mu)+E(\lambda, \mu)$.

Similar to the derivation from $(2.4 \mathrm{a}-2.4 \mathrm{~b})$ to $(2.5 \mathrm{a}-2.5 \mathrm{~b})$, we can establish from $(4.2 \mathrm{a}-4.2 \mathrm{~b})$ the following simplified independent recurrence relations

$$
\begin{aligned}
& x_{1+n}=2 u x_{n}-x_{n-1}+\frac{4-\lambda}{2-\lambda}(1-u) \\
& y_{1+n}=2 u y_{n}-y_{n-1}+\frac{4-\mu}{2-\mu}(1-u) .
\end{aligned}
$$

4.5. Generating functions. For an equivalent class of solutions satisfying the crossing recurrence relations $(4.2 \mathrm{a}-4.2 \mathrm{~b})$ with initial solutions $\left(x_{0}, y_{0}\right)$, let us denote the generating functions (cf. [9, §1.3-1.4]) by

$$
f(x):=\sum_{n=0}^{\infty} x^{n} x_{n} \quad \text { and } \quad g(y):=\sum_{n=0}^{\infty} y^{n} y_{n} .
$$


Multiplying (4.2a-4.2b) by $z^{n}$ and then performing the summation with respect to $n$ with $1 \leq n<\infty$, we get the following simplified functional equations

$$
\begin{aligned}
(1-u z) f(z)-H(\lambda, \mu) v z g(z) & =\frac{x_{0}+\left\{E(\lambda, \mu)-x_{0}\right\} z}{1-z} \\
(1-u z) g(z)-H(\mu, \lambda) v z g(z) & =\frac{y_{0}+\left\{E(\mu, \lambda)-y_{0}\right\} z}{1-z}
\end{aligned}
$$

Resolving this system of equations, we establish two generating functions

$$
\begin{aligned}
& f(x)=\frac{(1-u x)\left\{x_{0}-x x_{0}+x E(\lambda, \mu)\right\}+v x H(\lambda, \mu)\left\{y_{0}-x y_{0}+x E(\mu, \lambda)\right\}}{(1-x)\left(1-2 u x+x^{2}\right)} \\
& g(y)=\frac{(1-u y)\left\{y_{0}-y y_{0}+y E(\mu, \lambda)\right\}+v y H(\mu, \lambda)\left\{x_{0}-y x_{0}+y E(\lambda, \mu)\right\}}{(1-y)\left(1-2 u y+y^{2}\right)} .
\end{aligned}
$$

\section{Computer Algebra And Examples}

For two natural numbers $(\lambda, \mu)$ with $\lambda \neq \mu \geq 3$ and $(\lambda-2)(\mu-2)$ being a non perfect square number, the following procedure will be carried out in order to determine the recurrence relations, compute the generating functions and therefore to resolve the problem concerning the regular $(\lambda, \mu)$-polygonal numbers.

A. Write down the Diophantine equation (1.4) on the regular $(\lambda, \mu)$-polygonal numbers.

B. Figure out the generalized Pell equation $U^{2}-D(\lambda, \mu) V^{2}=N(\lambda, \mu)$ and the linear transformation $\Omega[\lambda, \mu]$.

C. Resolve the associated Pell equation $u^{2}-D(\lambda, \mu) v^{2}=1$ by PQ-algorithm (cf. $[4, \S 8.1]$ and $[8, \S 7.9]$.

D. Find out the minimum $(u, v)$ such that the crossing recurrence relations (4.2a-4.2b) have integer coefficients.

E. Determine the number of classes of equivalent solutions and initial values for each class through Brute-force search $([4, \S 8.3]$ and $[6, \S 5.3])$ and LMM-algorithm [5].

F. Exhibit crossing recurrence relations (4.2a-4.2b) of the first degree and independent recursions of the second degree (4.4a-4.4b).

G. Display explicitly generating functions (4.5a-4.5b) for each equivalent class of solutions.

In order to realize this procedure, a Mathematica package has been developed based on the theoretical preparation of the previous sections. It can provide us the necessary information about the regular $(\lambda, \mu)$-polygonal numbers. For the limit of space, here we present only a collection of the first twenty examples for $3 \leq \lambda \neq \mu \leq 9$ with $(\lambda-2)(\mu-2)$ being non perfect number, i.e. $(\lambda, \mu) \neq(3,6)$.

Example 1. Triangular and square numbers: 
- Diophantine equation: $x(1+x)=2 y^{2}$.

- Pell equation: $u^{2}-2 v^{2}=1$ where $u=1+2 x \& v=2 y$.

- Recurrences with initial condition $(1,1)$ :

$$
\left.\begin{array}{l}
x_{1+m}=3 x_{m}+4 y_{m}+1 \\
y_{1+m}=3 y_{m}+2 x_{m}+1
\end{array}\right\} \quad \text { and } \quad\left\{\begin{array}{l}
x_{1+n}=6 x_{n}-x_{n-1}+2 \\
y_{1+n}=6 y_{n}-y_{n-1}-0 .
\end{array}\right.
$$

- Generating functions:

$$
f(x)=\frac{1+x}{(1-x)\left(1-6 x+x^{2}\right)} \quad \text { and } \quad g(y)=\frac{1}{1-6 y+y^{2}} .
$$

- The first five numbers:

\begin{tabular}{||c|c|c|c||}
\hline \hline$n$ & $x_{n}$ & $y_{n}$ & $z_{n}$ \\
\hline 1 & 8 & 6 & 36 \\
\hline 2 & 49 & 35 & 1225 \\
\hline 3 & 288 & 204 & 41616 \\
\hline 4 & 1681 & 1189 & 1413721 \\
\hline 5 & 9800 & 6930 & 48024900 \\
\hline \hline
\end{tabular}

Example 2. Triangular and pentagonal numbers:

- Diophantine equation: $x(1+x)=y(3 y-1)$.

- Pell equation: $u^{2}-3 v^{2}=6$ where $u=3+6 x \& v=6 y-1$.

- Recurrences with initial condition $(1,1)$ :

$$
\left.\begin{array}{l}
x_{1+m}=7 x_{m}+12 y_{m}+1 \\
y_{1+m}=7 y_{m}+4 x_{m}+1
\end{array}\right\} \quad \text { and } \quad\left\{\begin{array}{l}
x_{1+n}=14 x_{n}-x_{n-1}+6 \\
y_{1+n}=14 y_{n}-y_{n-1}-2 .
\end{array}\right.
$$

- Generating functions:

$$
f(x)=\frac{1+5 x}{(1-x)\left(1-14 x+x^{2}\right)} \quad \text { and } \quad g(y)=\frac{1-3 y}{(1-y)\left(1-14 y+y^{2}\right)} .
$$

- The first five numbers:

\begin{tabular}{||c|c|c|c||}
\hline \hline$n$ & $x_{n}$ & $y_{n}$ & $z_{n}$ \\
\hline 1 & 20 & 12 & 210 \\
\hline 2 & 285 & 165 & 40755 \\
\hline 3 & 3976 & 2296 & 7906276 \\
\hline 4 & 55385 & $319^{r} 7$ & $1533^{r n} 76805$ \\
\hline 5 & 771420 & 445380 & 297544793910 \\
\hline \hline
\end{tabular}

Example 3. Pentagonal and square numbers:

- Diophantine equation: $x(3 x-1)=2 y^{2}$.

- Pell equation: $u^{2}-6 v^{2}=1$ where $u=6 x-1 \& v=2 y$.

- Recurrences with initial condition $(1,1)$ :

$$
\left.\begin{array}{l}
x_{1+m}=49 x_{m}+40 y_{m}-8 \\
y_{1+m}=49 y_{m}+60 x_{m}-10
\end{array}\right\} \quad \text { and } \quad\left\{\begin{array}{l}
x_{1+n}=98 x_{n}-x_{n-1}-16 \\
y_{1+n}=98 y_{n}-y_{n-1}-0 .
\end{array}\right.
$$


- Generating functions:

$$
f(x)=\frac{1-18 x+x^{2}}{(1-x)\left(1-98 x+x^{2}\right)} \quad \text { and } \quad g(y)=\frac{1-y^{2}}{(1-y)\left(1-98 y+y^{2}\right)} .
$$

- The first five numbers:

\begin{tabular}{||c|c|c|c||}
\hline \hline$n$ & $x_{n}$ & $y_{n}$ & $z_{n}$ \\
\hline 1 & 81 & 99 & 9801 \\
\hline 2 & 7921 & 9701 & 94109401 \\
\hline 3 & 776161 & 950599 & 903638458801 \\
\hline 4 & 76055841 & 93149001 & 8676736387998001 \\
\hline 5 & 7452696241 & 9127651499 & 83314021887196947001 \\
\hline \hline
\end{tabular}

Example 4. Hexagonal and square numbers:

- Diophantine equation: $2 x(2 x-1)=2 y^{2}$.

- Pell equation: $u^{2}-2 v^{2}=1$ where $u=4 x-1 \& v=2 y$.

- Recurrences with initial condition $(1,1)$ :

$$
\left.\begin{array}{l}
x_{1+m}=17 x_{m}+12 y_{m}-4 \\
y_{1+m}=17 y_{m}+24 x_{m}-6
\end{array}\right\} \quad \text { and } \quad\left\{\begin{array}{l}
x_{1+n}=34 x_{n}-x_{n-1}-8 \\
y_{1+n}=34 y_{n}-y_{n-1}-0 .
\end{array}\right.
$$

- Generating functions:

$$
f(x)=\frac{1-10 x+x^{2}}{(1-x)\left(1-34 x+x^{2}\right)} \quad \text { and } \quad g(y)=\frac{1-y^{2}}{(1-y)\left(1-34 y+y^{2}\right)} .
$$

- The first five numbers:

\begin{tabular}{||c|c|c|c||}
\hline \hline$n$ & $x_{n}$ & $y_{n}$ & $z_{n}$ \\
\hline 1 & 25 & 35 & 1225 \\
\hline 2 & 841 & 1189 & 1413721 \\
\hline 3 & 28561 & 40391 & 1631432881 \\
\hline 4 & 970225 & 1372105 & 1882672131025 \\
\hline 5 & 32959081 & 46611179 & 2172602007770041 \\
\hline \hline
\end{tabular}

Example 5. Regular (6,5)-polygonal numbers:

- Diophantine equation: $2 x(2 x-1)=y(3 y-1)$.

- Pell equation: $u^{2}-3 v^{2}=6$ where $u=3(4 x-1) \& v=6 y-1$.

- Recurrences with initial condition $(1,1)$ :

$$
\left.\begin{array}{l}
x_{1+m}=97 x_{m}+84 y_{m}-38 \\
y_{1+m}=97 y_{m}+112 x_{m}-44
\end{array}\right\} \quad \text { and } \quad\left\{\begin{array}{l}
x_{1+n}=194 x_{n}-x_{n-1}-48 \\
y_{1+n}=194 y_{n}-y_{n-1}-32 .
\end{array}\right.
$$

- Generating functions:

$$
f(x)=\frac{1-52 x+3 x^{2}}{(1-x)\left(1-194 x+x^{2}\right)} \quad \text { and } \quad g(y)=\frac{1-30 y-3 y^{2}}{(1-y)\left(1-194 y+y^{2}\right)} .
$$


- The first five numbers:

\begin{tabular}{||c|c|c|c||}
\hline \hline$n$ & $x_{n}$ & $y_{n}$ & $z_{n}$ \\
\hline 1 & 143 & 165 & 40755 \\
\hline 2 & 27693 & 31977 & 1533776805 \\
\hline 3 & 5372251 & 6203341 & 57722156241751 \\
\hline 4 & 1042188953 & 1203416145 & 2172315626468283465 \\
\hline 5 & 202179284583 & 233456528757 & 81752926228785223683195 \\
\hline \hline
\end{tabular}

Example 6. Regular (7,3)-polygonal numbers:

- Diophantine equation: $x(5 x-3)=y(1+y)$.

- Pell equation: $u^{2}-5 v^{2}=4$ where $u=10 x-3 \& v=1+2 y$.

- Recurrences with initial conditions $(1,1)$ and $(5,10)$ :

$$
\left.\begin{array}{l}
x_{1+m}=161 x_{m}+72 y_{m}-12 \\
y_{1+m}=161 y_{m}+360 x_{m}-28
\end{array}\right\} \quad \text { and } \quad\left\{\begin{array}{l}
x_{1+n}=322 x_{n}-x_{n-1}-96 \\
y_{1+n}=322 y_{n}-y_{n-1}+160
\end{array}\right.
$$

- Generating functions:

$$
\begin{aligned}
& f(x)=\frac{3}{10(1-x)}+\frac{1-x}{2\left(1-18 x+x^{2}\right)}+\frac{1-x}{5\left(1+18 x+x^{2}\right)} \\
& g(y)=\frac{-1}{2(1-y)}+\frac{1+y}{1-18 y+y^{2}}+\frac{1+y}{2\left(1+18 y+y^{2}\right)} .
\end{aligned}
$$

- The first five numbers:

\begin{tabular}{||c|c|c|c||}
\hline \hline$n$ & $x_{n}$ & $y_{n}$ & $z_{n}$ \\
\hline 1 & 5 & 10 & 55 \\
\hline 2 & 221 & 493 & 121771 \\
\hline 3 & 1513 & 3382 & 5720653 \\
\hline 4 & 71065 & 158905 & 12625478965 \\
\hline 5 & 487085 & 1089154 & 593128762435 \\
\hline \hline
\end{tabular}

Example 7. Regular $(7,4)$-polygonal numbers:

- Diophantine equation: $x(5 x-3)=2 y^{2}$.

- Pell equation: $u^{2}-10 v^{2}=9$ where $u=10 x-3 \& v=2 y$.

- Recurrences with initial conditions $(1,1),(6,9)$ and $(49,77)$ :

$$
\left.\begin{array}{l}
x_{1+m}=721 x_{m}+456 y_{m}-216 \\
y_{1+m}=721 y_{m}+1140 x_{m}-342
\end{array}\right\} \text { and }\left\{\begin{array}{l}
x_{1+n}=1442 x_{n}-x_{n-1}-432 \\
y_{1+n}=1442 y_{n}-y_{n-1}+0 .
\end{array}\right.
$$

- Generating functions:

$$
\begin{aligned}
& f(x)=\frac{3}{10(1-x)}+\frac{(1-x)\left(7+64 x+551 x^{2}+64 x^{3}+7 x^{4}\right)}{10\left(1-1442 x^{3}+x^{6}\right)} \\
& g(y)=\frac{(1+y)\left(1+8 y+69 y^{2}+8 y^{3}+y^{4}\right)}{1-1442 y^{3}+y^{6}} .
\end{aligned}
$$


- The first five numbers:

\begin{tabular}{||c|c|c|c||}
\hline \hline$n$ & $x_{n}$ & $y_{n}$ & $z_{n}$ \\
\hline 1 & 6 & 9 & 81 \\
\hline 2 & 49 & 77 & 5929 \\
\hline 3 & 961 & 1519 & 2307361 \\
\hline 4 & 8214 & 12987 & 168662169 \\
\hline 5 & 70225 & 111035 & 12328771225 \\
\hline \hline
\end{tabular}

Example 8. Regular $(7,5)$-polygonal numbers:

- Diophantine equation: $x(5 x-3)=y(3 y-1)$.

- Pell equation: $u^{2}-15 v^{2}=66$ where $u=3(10 x-3) \& v=6 y-1$.

- Recurrences with initial condition $(1,1)$ :

$$
\left.\begin{array}{l}
x_{1+m}=31 x_{m}+24 y_{m}-13 \\
y_{1+m}=31 y_{m}+40 x_{m}-17
\end{array}\right\} \quad \text { and } \quad\left\{\begin{array}{l}
x_{1+n}=62 x_{n}-x_{n-1}-18 \\
y_{1+n}=62 y_{n}-y_{n-1}-10 .
\end{array}\right.
$$

- Generating functions:

$$
f(x)=\frac{1-21 x+2 x^{2}}{(1-x)\left(1-62 x+x^{2}\right)} \quad \text { and } \quad g(y)=\frac{1-9 y-2 y^{2}}{(1-y)\left(1-62 y+y^{2}\right)} .
$$

- The first five numbers:

\begin{tabular}{||c|c|c|c||}
\hline \hline$n$ & $x_{n}$ & $y_{n}$ & $z_{n}$ \\
\hline 1 & 42 & 54 & 4347 \\
\hline 2 & 2585 & 3337 & 16701685 \\
\hline 3 & 160210 & 206830 & 64167869935 \\
\hline 4 & 9930417 & 12820113 & 246532939589097 \\
\hline 5 & 615525626 & 794640166 & 947179489733441251 \\
\hline \hline
\end{tabular}

Example 9. Regular (7,6)-polygonal numbers:

- Diophantine equation: $x(5 x-3)=2 y(2 y-1)$.

- Pell equation: $u^{2}-5 v^{2}=4$ where $u=10 x-3 \& v=4 y-1$.

- Recurrences with initial condition $(1,1)$.

$$
\left.\begin{array}{l}
x_{1+m}=161 x_{m}+144 y_{m}-84 \\
y_{1+m}=161 y_{m}+180 x_{m}-94
\end{array}\right\} \text { and }\left\{\begin{array}{l}
x_{1+n}=322 x_{n}-x_{n-1}-96 \\
y_{1+n}=322 y_{n}-y_{n-1}-80 .
\end{array}\right.
$$

- Generating functions:

$$
f(x)=\frac{1-102 x+5 x^{2}}{(1-x)\left(1-322 x+x^{2}\right)} \quad \text { and } \quad g(y)=\frac{1-76 y-5 y^{2}}{(1-y)\left(1-322 y+y^{2}\right)} \text {. }
$$


- The first five numbers:

\begin{tabular}{||c|c|c|c||}
\hline \hline$n$ & $x_{n}$ & $y_{n}$ & $z_{n}$ \\
\hline 1 & 221 & 247 & 121771 \\
\hline 2 & 71065 & 79453 & 12625478965 \\
\hline 3 & 22882613 & 25583539 & 1309034909945503 \\
\hline 4 & 7368130225 & 8237820025 & 135723357520344181255 \\
\hline 5 & 2372515049741 & 2652552464431 & 14072069153115290487843091 \\
\hline \hline
\end{tabular}

Example 10. Regular (8,3)-polygonal numbers:

- Diophantine equation: $2 x(3 x-2)=y(1+y)$.

- Pell equation: $u^{2}-6 v^{2}=10$ where $u=4(3 x-1) \& v=1+2 y$.

- Recurrences with initial conditions $(1,1)$ and $(3,6)$ :

$$
\left.\begin{array}{l}
x_{1+m}=49 x_{m}+20 y_{m}-6 \\
y_{1+m}=49 y_{m}+120 x_{m}-16
\end{array}\right\} \quad \text { and } \quad\left\{\begin{array}{l}
x_{1+n}=98 x_{n}-x_{n-1}-32 \\
y_{1+n}=98 y_{n}-y_{n-1}+48
\end{array}\right.
$$

- Generating functions:

$$
\begin{aligned}
& f(x)=\frac{1}{3(1-x)}+\frac{1-x}{2\left(1-10 x+x^{2}\right)}+\frac{1-x}{6\left(1+10 x+x^{2}\right)} \\
& g(y)=\frac{-1}{2(1-y)}+\frac{1+y}{1-10 y+y^{2}}+\frac{1+y}{2\left(1+10 y+y^{2}\right)} .
\end{aligned}
$$

- The first five numbers:

\begin{tabular}{||c|c|c|c||}
\hline \hline$n$ & $x_{n}$ & $y_{n}$ & $z_{n}$ \\
\hline 1 & 3 & 6 & 21 \\
\hline 2 & 63 & 153 & 11781 \\
\hline 3 & 261 & 638 & 203841 \\
\hline 4 & 6141 & 15041 & 113123361 \\
\hline 5 & 25543 & 62566 & 1957283461 \\
\hline \hline
\end{tabular}

Example 11. Regular (8,4)-polygonal numbers:

- Diophantine equation: $2 x(3 x-2)=2 y^{2}$.

- Pell equation: $u^{2}-3 v^{2}=1$ where $u=3 x-1 \& v=y$.

- Recurrences with initial condition $(1,1)$ :

$$
\left.\begin{array}{l}
x_{1+m}=7 x_{m}+4 y_{m}-2 \\
y_{1+m}=7 y_{m}+12 x_{m}-4
\end{array}\right\} \quad \text { and } \quad\left\{\begin{array}{l}
x_{1+n}=14 x_{n}-x_{n-1}-4 \\
y_{1+n}=14 y_{n}-y_{n-1}-0
\end{array}\right.
$$

- Generating functions:

$$
f(x)=\frac{1-6 x+x^{2}}{(1-x)\left(1-14 x+x^{2}\right)} \quad \text { and } \quad g(y)=\frac{1-y^{2}}{(1-y)\left(1-14 y+y^{2}\right)} .
$$


- The first five numbers:

\begin{tabular}{||c|c|c|c||}
\hline \hline$n$ & $x_{n}$ & $y_{n}$ & $z_{n}$ \\
\hline 1 & 9 & 15 & 225 \\
\hline 2 & 121 & 209 & 43681 \\
\hline 3 & 1681 & 2911 & 8473921 \\
\hline 4 & 23409 & 40545 & 1643897025 \\
\hline 5 & 326041 & 564719 & 318907548961 \\
\hline \hline
\end{tabular}

Example 12. Regular (8,5)-polygonal numbers:

- Diophantine equation: $2 x(3 x-2)=y(3 y-1)$.

- Pell equation: $u^{2}-2 v^{2}=14$ where $u=4(3 x-1) \& v=6 y-1$.

- Recurrences with initial conditions $(1,1)$ and $(8,11)$ :

$$
\left.\begin{array}{l}
x_{1+m}=577 x_{m}+408 y_{m}-260 \\
y_{1+m}=577 y_{m}+816 x_{m}-368
\end{array}\right\} \text { and }\left\{\begin{array}{l}
x_{1+n}=1154 x_{n}-x_{n-1}-384 \\
y_{1+n}=1154 y_{n}-y_{n-1}-192 .
\end{array}\right.
$$

- Generating functions:

$$
\begin{aligned}
& f(x)=\frac{1}{3(1-x)}+\frac{1-5 x}{2\left(1-34 x+x^{2}\right)}+\frac{1-7 x}{6\left(1+34 x+x^{2}\right)} \\
& g(y)=\frac{1}{6(1-y)}+\frac{1+7 y}{2\left(1-34 y+y^{2}\right)}+\frac{1+5 y}{3\left(1+34 y+y^{2}\right)}
\end{aligned}
$$

- The first five numbers:

\begin{tabular}{||c|c|c|c||}
\hline \hline$n$ & $x_{n}$ & $y_{n}$ & $z_{n}$ \\
\hline 1 & 8 & 11 & 176 \\
\hline 2 & 725 & 1025 & 1575425 \\
\hline 3 & 8844 & 12507 & 234631320 \\
\hline 4 & 836265 & 1182657 & 2098015778145 \\
\hline 5 & 10205584 & 14432875 & 312461813932000 \\
\hline \hline
\end{tabular}

Example 13. Regular (8,6)-polygonal numbers:

- Diophantine equation: $2 x(3 x-2)=2 y(2 y-1)$.

- Pell equation: $u^{2}-6 v^{2}=10$ where $u=4(3 x-1) \& v=4 y-1$.

- Recurrences with initial condition $(1,1)$ :

$$
\left.\begin{array}{l}
x_{1+m}=49 x_{m}+40 y_{m}-26 \\
y_{1+m}=49 y_{m}+60 x_{m}-32
\end{array}\right\} \quad \text { and } \quad\left\{\begin{array}{l}
x_{1+n}=98 x_{n}-x_{n-1}-32 \\
y_{1+n}=98 y_{n}-y_{n-1}-24 .
\end{array}\right.
$$

- Generating functions:

$$
f(x)=\frac{1-36 x+3 x^{2}}{(1-x)\left(1-98 x+x^{2}\right)} \quad \text { and } \quad g(y)=\frac{1-22 y-3 y^{2}}{(1-y)\left(1-98 y+y^{2}\right)} .
$$


- The first five numbers:

\begin{tabular}{||c|c|c|c||}
\hline \hline$n$ & $x_{n}$ & $y_{n}$ & $z_{n}$ \\
\hline 1 & 63 & 77 & 11781 \\
\hline 2 & 6141 & 7521 & 113123361 \\
\hline 3 & 601723 & 736957 & 1086210502741 \\
\hline 4 & 58962681 & 72214241 & 10429793134197921 \\
\hline 5 & 5777740983 & 7076258637 & 100146872588357936901 \\
\hline \hline
\end{tabular}

Example 14. Regular $(8,7)$-polygonal numbers:

- Diophantine equation: $2 x(3 x-2)=y(5 y-3)$.

- Pell equation: $u^{2}-30 v^{2}=130$ where $u=20(3 x-1) \& v=10 y-3$.

- Recurrences with initial condition $(1,1)$ :

$$
\left.\begin{array}{l}
x_{1+m}=241 x_{m}+220 y_{m}-146 \\
y_{1+m}=241 y_{m}+264 x_{m}-160
\end{array}\right\} \text { and }\left\{\begin{array}{l}
x_{1+n}=482 x_{n}-x_{n-1}-160 \\
y_{1+n}=482 y_{n}-y_{n-1}-144 .
\end{array}\right.
$$

- Generating functions:

$$
f(x)=\frac{1-168 x+7 x^{2}}{(1-x)\left(1-482 x+x^{2}\right)} \quad \text { and } \quad g(y)=\frac{1-138 y-7 y^{2}}{(1-y)\left(1-482 y+y^{2}\right)} \text {. }
$$

- The first five numbers:

\begin{tabular}{||c|c|c|c||}
\hline \hline$n$ & $x_{n}$ & $y_{n}$ & $z_{n}$ \\
\hline 1 & 315 & 345 & 297045 \\
\hline 2 & 151669 & 166145 & 69010153345 \\
\hline 3 & 73103983 & 80081401 & 16032576845184901 \\
\hline 4 & 35235967977 & 38599068993 & 3724720317758036481633 \\
\hline 5 & 16983663460771 & 18604671173081 & 865334473646149974640821781 \\
\hline \hline
\end{tabular}

Example 15. Regular (9,3)-polygonal numbers:

- Diophantine equation: $x(7 x-5)=y(1+y)$.

- Pell equation: $u^{2}-7 v^{2}=18$ where $u=14 x-5 \& v=1+2 y$.

- Recurrences with initial condition $(1,1)$ :

$$
\left.\begin{array}{l}
x_{1+m}=8 x_{m}+3 y_{m}-1 \\
y_{1+m}=8 y_{m}+21 x_{m}-4
\end{array}\right\} \quad \text { and } \quad\left\{\begin{array}{l}
x_{1+n}=16 x_{n}-x_{n-1}-5 \\
y_{1+n}=16 y_{n}-y_{n-1}+7 .
\end{array}\right.
$$

- Generating functions:

$$
f(x)=\frac{1-7 x+x^{2}}{(1-x)\left(1-16 x+x^{2}\right)} \quad \text { and } \quad g(y)=\frac{1+8 y-2 y^{2}}{(1-y)\left(1-16 y+y^{2}\right)} .
$$


- The first five numbers:

\begin{tabular}{||c|c|c|c||}
\hline \hline$n$ & $x_{n}$ & $y_{n}$ & $z_{n}$ \\
\hline 1 & 10 & 25 & 325 \\
\hline 2 & 154 & 406 & 82621 \\
\hline 3 & 2449 & 6478 & 20985481 \\
\hline 4 & 39025 & 103249 & 5330229625 \\
\hline 5 & 621946 & 1645513 & 1353857339341 \\
\hline \hline
\end{tabular}

Example 16. Regular $(9,4)$-polygonal numbers:

- Diophantine equation: $x(7 x-5)=2 y^{2}$.

- Pell equation: $u^{2}-14 v^{2}=25$ where $u=14 x-5 \& v=2 y$.

- Recurrences with initial conditions $(1,1)$ and $(2,3)$ :

$$
\left.\begin{array}{l}
x_{1+m}=15 x_{m}+8 y_{m}-5 \\
y_{1+m}=15 y_{m}+28 x_{m}-10
\end{array}\right\} \quad \text { and } \quad\left\{\begin{array}{l}
x_{1+n}=30 x_{n}-x_{n-1}-10 \\
y_{1+n}=30 y_{n}-y_{n-1}+0 .
\end{array}\right.
$$

- Generating functions:

$$
\begin{aligned}
& f(x)=\frac{5}{14(1-x)}+\frac{(1-x)\left(9+32 x+9 x^{2}\right)}{14\left(1-30 x^{2}+x^{4}\right)} \\
& g(y)=\frac{(1+y)^{3}}{1-30 y^{2}+y^{4}} .
\end{aligned}
$$

- The first five numbers:

\begin{tabular}{||c|c|c|c||}
\hline \hline$n$ & $x_{n}$ & $y_{n}$ & $z_{n}$ \\
\hline 1 & 2 & 3 & 9 \\
\hline 2 & 18 & 33 & 1089 \\
\hline 3 & 49 & 91 & 8281 \\
\hline 4 & 529 & 989 & 978121 \\
\hline 5 & 1458 & 2727 & 7436529 \\
\hline \hline
\end{tabular}

Example 17. Regular (9,5)-polygonal numbers:

- Diophantine equation: $x(7 x-5)=y(3 y-1)$.

- Pell equation: $u^{2}-21 v^{2}=204$ where $u=3(14 x-5) \& v=6 y-1$.

- Recurrences with initial conditions $(1,1)$ and $(14,21)$ :

$\left.\begin{array}{l}x_{1+m}=6049 x_{m}+3960 y_{m}-2820 \\ y_{1+m}=6049 y_{m}+9240 x_{m}-4308\end{array}\right\}$ and $\left\{\begin{array}{l}x_{1+n}=12098 x_{n}-x_{n-1}-4320 \\ y_{1+n}=12098 y_{n}-y_{n-1}-2016 .\end{array}\right.$

- Generating functions:

$$
\begin{aligned}
& f(x)=\frac{5}{14(1-x)}+\frac{1-31 x}{2\left(1-110 x+x^{2}\right)}+\frac{1-71 x}{7\left(1+110 x+x^{2}\right)} \\
& g(y)=\frac{1}{6(1-y)}+\frac{1+71 y}{3\left(1-110 y+y^{2}\right)}+\frac{1+31 y}{2\left(1+110 y+y^{2}\right)} .
\end{aligned}
$$


- The first five numbers:

\begin{tabular}{||c|c|c|c||}
\hline \hline$n$ & $x_{n}$ & $y_{n}$ & $z_{n}$ \\
\hline 1 & 14 & 21 & 651 \\
\hline 2 & 7189 & 10981 & 180868051 \\
\hline 3 & 165026 & 252081 & 95317119801 \\
\hline 4 & 86968201 & 132846121 & 26472137730696901 \\
\hline 5 & 1996480214 & 3049673901 & 13950766352135999751 \\
\hline \hline
\end{tabular}

Example 18. Regular (9,6)-polygonal numbers:

- Diophantine equation: $x(7 x-5)=2 y(2 y-1)$.

- Pell equation: $u^{2}-7 v^{2}=18$ where $u=14 x-5 \& v=4 y-1$.

- Recurrences with initial conditions $(1,1)$ and $(10,13)$ :

$\left.\begin{array}{l}x_{1+m}=32257 x_{m}+24384 y_{m}-17616 \\ y_{1+m}=32257 y_{m}+42672 x_{m}-23304\end{array}\right\}$ and $\left\{\begin{array}{l}x_{1+n}=64514 x_{n}-x_{n-1}-23040 \\ y_{1+n}=64514 y_{n}-y_{n-1}-16128\end{array}\right.$

- Generating functions:

$$
\begin{aligned}
& f(x)=\frac{5}{14(1-x)}+\frac{9(1-127 x)}{14\left(1-254 x+x^{2}\right)}-\frac{72 x}{1+254 x+x^{2}} \\
& g(y)=\frac{1}{4(1-y)}+\frac{108 y}{1-254 y+y^{2}}+\frac{3(1+127 y)}{4\left(1+254 y+y^{2}\right)} .
\end{aligned}
$$

- The first five numbers:

\begin{tabular}{||c|c|c|c||}
\hline \hline$n$ & $x_{n}$ & $y_{n}$ & $z_{n}$ \\
\hline 1 & 10 & 13 & 325 \\
\hline 2 & 39025 & 51625 & 5330229625 \\
\hline 3 & 621946 & 822757 & 1353857339341 \\
\hline 4 & 2517635809 & 3330519121 & $2218471522^{7} 7362706161$ \\
\hline 5 & 40124201194 & 53079328957 & 5634830324997758086741 \\
\hline \hline
\end{tabular}

Example 19. Regular $(9,7)$-polygonal numbers:

- Diophantine equation: $x(7 x-5)=y(5 y-3)$.

- Pell equation: $u^{2}-35 v^{2}=310$ where $u=5(14 x-5) \& v=10 y-3$.

- Recurrences with initial condition $(1,1)$.

$$
\left.\begin{array}{l}
x_{1+m}=71 x_{m}+60 y_{m}-43 \\
y_{1+m}=71 y_{m}+84 x_{m}-51
\end{array}\right\} \quad \text { and } \quad\left\{\begin{array}{l}
x_{1+n}=142 x_{n}-x_{n-1}-50 \\
y_{1+n}=142 y_{n}-y_{n-1}-42
\end{array}\right.
$$

- Generating functions:

$$
f(x)=\frac{1-55 x+4 x^{2}}{(1-x)\left(1-142 x+x^{2}\right)} \quad \text { and } \quad g(y)=\frac{1-39 y-4 y^{2}}{(1-y)\left(1-142 y+y^{2}\right)} .
$$


- The first five numbers:

\begin{tabular}{||c|c|c|c||}
\hline \hline$n$ & $x_{n}$ & $y_{n}$ & $z_{n}$ \\
\hline 1 & 88 & 104 & 26884 \\
\hline 2 & 12445 & 14725 & 542041975 \\
\hline 3 & 1767052 & 2090804 & 10928650279834 \\
\hline 4 & 250908889 & 296879401 & 220343446399977901 \\
\hline 5 & 35627295136 & 42154784096 & 4442564555387704166896 \\
\hline \hline
\end{tabular}

Example 20. Regular (9,8)-polygonal numbers:

- Diophantine equation: $x(7 x-5)=2 y(3 y-2)$.

- Pell equation: $u^{2}-42 v^{2}=57$ where $u=3(14 x-5) \& v=2(3 y-1)$.

- Recurrences with initial condition $(1,1)$ :

$$
\left.\begin{array}{l}
x_{1+m}=337 x_{m}+312 y_{m}-224 \\
y_{1+m}=337 y_{m}+364 x_{m}-242
\end{array}\right\} \quad \text { and } \quad\left\{\begin{array}{l}
x_{1+n}=674 x_{n}-x_{n-1}-240 \\
y_{1+n}=674 y_{n}-y_{n-1}-224 .
\end{array}\right.
$$

- Generating functions:

$$
f(x)=\frac{1-250 x+9 x^{2}}{(1-x)\left(1-674 x+x^{2}\right)} \quad \text { and } \quad g(y)=\frac{1-216 y-9 y^{2}}{(1-y)\left(1-674 y+y^{2}\right)} \text {. }
$$

- The first five numbers:

\begin{tabular}{||c|c|c|c||}
\hline \hline$n$ & $x_{n}$ & $y_{n}$ & $z_{n}$ \\
\hline 1 & 425 & 459 & 631125 \\
\hline 2 & 286209 & 309141 & 286703855361 \\
\hline 3 & 192904201 & 208360351 & 130242107189808901 \\
\hline 4 & 130017145025 & 140434567209 & 59165603001256545014625 \\
\hline 5 & 87631362842409 & 94652689938291 & 26877395137662573622784125461 \\
\hline \hline
\end{tabular}

\section{REFERENCES}

[1] L. E. Dickson, History of the Theory of Numbers: Volume II - Diophantine Analysis, New York Stechert, 1934.

[2] G. H. Hardy \& E. M. Wright, An Introduction to the Theory of Numbers, Oxford University Press, 1979.

[3] L. K. Hua, Introduction to the Theory of Numbers, Springer-Verlag, Berlin - New York, 1982.

[4] V. J. LeVeque, Topics in Number Theory, Addison-Wesley, New York, 1956.

[5] K. Matthews, The Diophantine equation $x^{2}-D y^{2}=N$, Expositiones Mathematicae 18 (2000), 323-331.

[6] R. E. Mollin, Fundamental Number Theory with Applications, CRC Press, Boca Raton, 1998.

[7] T. Nagell, Introduction to Number Theory, Chelsea Publishing Company, New York, 1981.

[8] I. Niven, H. S. Zuckerman e H. L. Montgomery, An Introduction to the Theory of Numbers, John Wiley \& Sons, New York, 1980.

[9] H. S. Wilf, Generatingfunctionology (second edition), Academic Press Inc., London, 1994.

Received: April 19, 2006 